\title{
Simulation of 3D laser scanning with phase-based EDM for the prediction of systematic deviations
}

\section{Conference Paper}

\section{Author(s):}

Chaudhry, Sukant; Salido-Monzú, David; Wieser, Andreas

Publication date:

2019-06-21

Permanent link:

https://doi.org/10.3929/ethz-b-000350320

Rights / license:

In Copyright - Non-Commercial Use Permitted

Originally published in:

11057, https://doi.org/10.1117/12.2526043

\section{Funding acknowledgement:}

169318 - Modeling surface-related uncertainty of terrestrial laser scanning (SNF) 


\section{Simulation of 3D laser scanning with phase-based EDM for the prediction of systematic deviations}

Sukant Chaudhry, David Salido-Monzú, Andreas Wieser

Sukant Chaudhry, David Salido-Monzú, Andreas Wieser, "Simulation of 3D laser scanning with phase-based EDM for the prediction of systematic deviations," Proc. SPIE 11057, Modeling Aspects in Optical Metrology VII, 110570H (21 June 2019); doi: 10.1117/12.2526043

SPIE. Event: SPIE Optical Metrology, 2019, Munich, Germany 


\title{
Simulation of 3d laser scanning with phase-based EDM for the prediction of systematic deviations
}

\author{
Sukant Chaudhry, David Salido-Monzú, and Andreas Wieser \\ Institute of Geodesy and Photogrammetry, ETH Zurich, Switzerland
}

\begin{abstract}
Reflectorless electro-optical distance measurement (RL-EDM) relies on measuring the round-trip time of optical signals transmitted from the instrument and reflected by natural surfaces. It is the backbone of laser scanning technology, which allows easily digitizing the environment and obtaining $3 \mathrm{~d}$ models that represent the geometry of the scanned objects. However, the measured distances do not refer to single, well-defined target points at the object surface but rather correspond to a weighted average of effective distances within the respective footprint of the laser beam. This increases the uncertainty of the measurements and may cause systematic deviations significantly exceeding the mm-or sub-mm level precision that would otherwise be attainable with EDM technology.

In this paper we introduce a numerical simulation of the measurement process for phase-based RL-EDM with I/Q-demodulation assuming a Gaussian beam profile. The beam is discretized into a fixed number of rays for each of which the corresponding phase delay and attenuation are calculated. The I- and Q-components are obtained by integration over the footprint taking the beam profile into account. By deflection of the beam into incrementally changed spatial directions we extend the simulation to one of the $3 \mathrm{~d}$ scanning process. The scanned surfaces are represented by triangular irregular meshes (TINs) with high spatial resolution. Each triangle is associated with a reflectivity, as a starting point for the modelling of surface properties. The simulation takes the interplay between the energy distribution within the laser footprint, the surface geometry and the surface reflectivity into account.

Herein, we use the simulation framework to study the effects of the angle of incidence, of surface curvature and of mixed pixels in absence of measurement noise. The results indicate that the angle of incidence at a planar surface and the surface curvature within the footprint are on the order of $0.1 \mathrm{~mm}$ or less for small footprint and angles of incidence below about $60 \mathrm{deg}$. If the footprint and the angle of incidence are very large the biases may reach mm-level, however even then the impact of measurement noise and surface roughness will typically exceed these biases, such that they are negligible. On the other hand we show using simulations and real scans of a cylinder in front of a planar background, that the impact of mixed pixels or beams only partially hitting an object may introduce large biases and is practically relevant.
\end{abstract}

Keywords: electronic distance measurement (EDM), laser scanning, numerical simulation, ray tracing modeling, systematic deviations, mixed pixels.

Further author information: (Send correspondence to S.C.)

S.C.: E-mail: sukant.chaudhry@geod.baug.ethz.ch, Telephone: +41446336642

D.S.M.: E-mail: david.salido@geod.baug.ethz.ch, Telephone: +414463342 26

A.W.: E-mail: andreas.wieser@geod.baug.ethz.ch, Telephone: +41 446330555

Modeling Aspects in Optical Metrology VII, edited by Bernd Bodermann, Karsten Frenner, Proc. of SPIE Vol. 11057, 110570H - @ 2019 SPIE - CCC code: 0277-786X/19/\$21 - doi: 10.1117/12.2526043 


\section{INTRODUCTION}

Terrestrial 3d laser scanning (TLS) is a widely used remote sensing technology that allows sampling the geometry and reflectivity of natural and man-made surfaces. The models derived from TLS are used in a variety of applications. ${ }^{1}$ However, insufficient knowledge of the uncertainties of the reflectorless electronic distance measurement (RL-EDM) carried out by the LiDAR unit of the scanner impairs widespread application when reliable prediction of accuracies at the $\mathrm{mm}$ to sub-mm level is required. The instrument specifications published by the manufacturers quantify the performance of the instrument itself, referring to standardized and reproducible measurement configurations in terms of distance, angle-of-incidence, surface type and reflectivity. They are not representative for practical use of the instrument. Surface-related uncertainties originating from the impact of roughness, variable reflectivities, multiple reflections, surface penetration and similar effects at natural surfaces often exceed the instrument specifications by far.

Schulz $^{2}$ has studied effects related to the type of object surface and to the angle of incidence (AOI). He investigated surface penetration effects for certain materials finding related distance biases of up to $15 \mathrm{~mm}$ for styrofoam and $5 \mathrm{~mm}$ for wood. A summary of various surface effects was given by Reshetyuk, ${ }^{3}$ indicating that the reflectivity is the key parameter controlling the impact of the surface on the measurement and listing various factors affecting the reflectance. Other authors reported experimental investigations indicating distance biases related to surface reflectivity, materials and AOI. ${ }^{4,5}$ Empirical models of distance noise as a function of AOI have been proposed, either using only the AOI, ${ }^{6}$ using the distance and the surface type additionally, ${ }^{7}$ or using variance component estimation. ${ }^{8}$ More recently, also an approach to quantify the uncertainty of the scanned distances using the signal intensity instead of the AOI has been published. ${ }^{9}$

The main reasons for the dependence of noise and biases on the AOI are the reduction of received signal power (and thus higher measurement noise) typically encountered with increasing AOI and the fact that the finite beam-width illuminates an increasingly larger area with increasing AOI. The achievable angular resolution during the scanning is usually limited primarily by the energy distribution within this area, i.e. within the socalled footprint of the laser beam, rather than by the minimum increment of horizontal and vertical angles for the beam deflection. ${ }^{10}$ So, surface roughness on scales larger than the footprint (because of the impact on AOI) and on scales smaller than the footprint (because the distance measurement effectively represents a weighted average of the distances within the footprint) has an impact on both noise and bias of the distance measurements. While many of the surface-related effects have been investigated individually and mostly empirically, a comprehensive model linking causes and effects, allowing prediction of deviations and identification of the main drivers of surface-related biases is not yet available.

In this paper, we present a numerical simulation framework of the LiDAR measurement process. This simulation is an important tool for the investigation of uncertainties in laser scanning and thus a basis for deriving a comprehensive error model at a later stage. TLS data are acquired by (i) estimating the delay of optical signals emitted by the scanner and reflected by the surface parts within the footprint, ${ }^{11}$ and (ii) incrementally deflecting the laser beam about two (orthogonal) axes. The simulation covers both aspects. However, the focus herein is on simulating phase-based EDM with I/Q-demodulation. ${ }^{12}$ We apply a purely geometrical and ray optics approach assuming a fundamental Gaussian laser beam, i.e., $\mathrm{TEM}_{00}$ laser mode, ${ }^{13}$ and consequently discretizing the beam with Gaussian profile spatially into a fixed number or rays each of which interacts with the surface and contributes to the measured distance.

We demonstrate the use of the simulation framework by studying (i) the systematic effects of the angle of incidence when scanning a perfectly planar and homogeneous surface from different distances, and (ii) the systematic effects of surface curvature and multiple reflecting surfaces at different distances within the same beam (mixed pixels) when scanning a cylinder. We compare the results of the simulation to real scans of a cylinder and use the results of this analysis to explain the deviations to be expected when fitting geometric primitives to such point clouds. While this investigation is a building block for the research towards a comprehensive error model of 3d laser scanning, it also has direct practical relevance by itself as indicated e.g., by the systematic overestimation of tree stem diameters extracted from point clouds reported. ${ }^{14}$

The following section gives a detailed summary of the phase-based RL-EDM measurement simulation with the key equations, and a short description of the laboratory experimental setup used for validating the simulations. 
Section 3 presents exemplary results of the application of the simulation framework to the two case studies mentioned above. It covers both qualitative and quantitative aspects, and is followed by the conclusions.

\section{METHODOLOGY}

The simulation framework relies on ray tracing of Gaussian beams decomposed into a fixed number of rays, and a model of the phase-based measurement process considering both radiometric and geometric aspects. It thus accounts for the impact of each ray on the final distance estimation, considering its interaction with the underlying surface properties. The underlying mathematical model includes the energy distribution within the discretized laser footprint, the surface geometry, and the reflectivity of the surface material. Within this paper, the simulations are complemented by an experimental set-up that enables validation and comparison using measurements obtained with a commercial laser scanner.

\subsection{Model of the phase-based RL-EDM measurement process}

The RL-EDM measurement is intended to yield an estimate of the distance between the instrument and the footprint $(\mathcal{F})$ of the scanning laser beam. However, since the footprint has finite dimension different surface points within the footprint have different distance from the instrument (see Figure 1). While pulse-based instruments capable of waveform-digitizing $\operatorname{LiDAR}^{15}$ may be able to distinguish some areas within the footprint if the distances are sufficiently different as compared to the pulse-width, phase-based instruments cannot distinguish them. The real measurement process can thus be interpreted as a weighted averaging of all the distances $d$ between the instrument and the surface within $\mathcal{F}$. The corresponding weights ( $\bar{p}$, see Eq. 1b) depend on the measurement process within the instrument, on the energy received after reflection at the corresponding surface point and possibly on further parameters. They are at least a function of the position of the point within the footprint, the beam profile, the wavelength, the distance, the angle of incidence, local surface properties like the roughness, the directional reflectivity and the materials.

We will later see that a more convenient general interpretation expresses the distance estimate as a functional of the distances and certain (other) weights $p$ comprising integration over the entire footprint (see Eq. 1a). In our case, $g(\cdot)$ will be a vector valued function of the scalar distances, and $f(\cdot)$ a scalar function of the vector valued integration results. We therefore write the estimated distance $\hat{D}$ formally as follows:

$$
\begin{aligned}
\hat{D} & =f\left(\iint_{\mathcal{F}} p(\theta, \phi) \cdot g(d(\theta, \phi)) d \theta d \phi\right), \\
& =\iint_{\mathcal{F}} \bar{p}(\theta, \phi) \cdot d(\theta, \phi) d \theta d \phi,
\end{aligned}
$$

where the footprint is expressed as the subspace of all horizontal angles $\theta$ and all vertical angles $\phi$ (see Figure 2) for which the irradiance on the surface is not negligible.

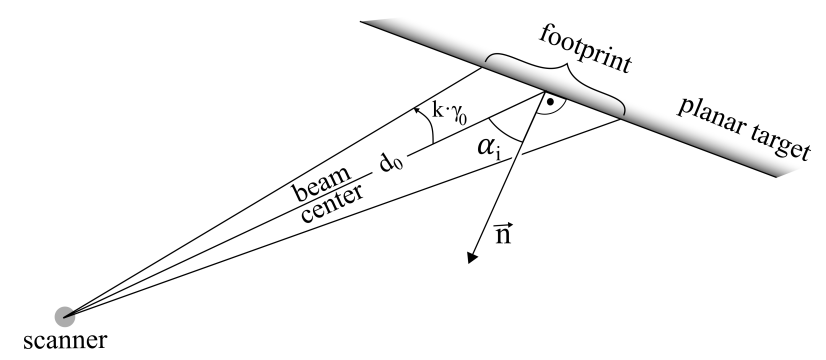

Figure 1: The measurement laser beam and the footprint defined by a solid angle $2\left(k \gamma_{0}\right)$, here indicated for a perfectly planar target.

We subsequently assume that the laser scanner produces the coordinates of scanned points within its own right-handed Cartesian coordinate system (the scanner's own coordinate system, SOCS), denoted $\left(X^{S}, Y^{S}, Z^{S}\right)$ 
in Figure 2, and that the scanner is perfectly calibrated such that the center axis of the laser beam contains the origin of the SOCS. At a specific point in time, the beam center points into a spatial direction described by the angles $\theta_{0}$ and $\phi_{0}$ within the SOCS. We define a right-handed Cartesian coordinate system $(\xi, \eta, \zeta)$ connected with the beam such that its $\zeta$-axis coincides with the beam center, its origin is at a certain distance $\zeta_{0}$ from the origin of the SOCS, and the $\eta$-axis is contained in the $\left(\zeta, Z^{S}\right)$-plane (vertical plane, if $Z^{S}$ is vertical). The transformation of coordinates between the two systems is then described by a rotation and a translation as follows:

$$
\left[\begin{array}{l}
X^{S} \\
Y^{S} \\
Z^{S}
\end{array}\right]=\left[\begin{array}{ccc}
\sin \theta_{0} & \cos \theta_{0} \sin \phi_{0} & \cos \theta_{0} \cos \phi_{0} \\
-\cos \theta_{0} & \sin \theta_{0} \sin \phi_{0} & \sin \theta_{0} \cos \phi_{0} \\
0 & -\cos \phi_{0} & \sin \phi_{0}
\end{array}\right] \cdot\left[\begin{array}{c}
\xi \\
\eta \\
\zeta+\zeta_{0}
\end{array}\right]
$$

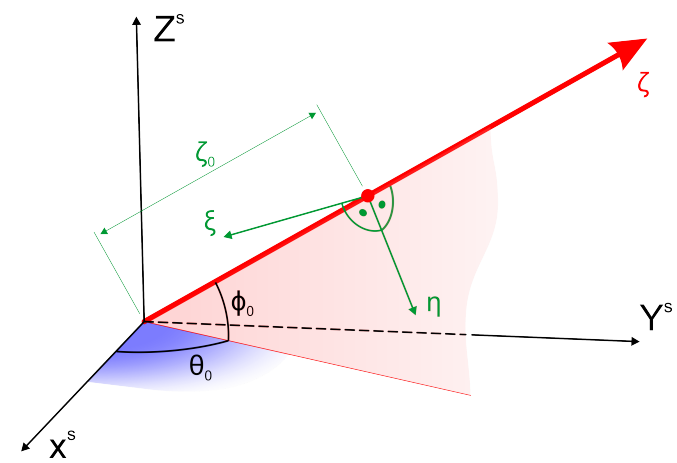

Figure 2: The laser scanner coordinate system and the local beam coordinate system for a particular beam direction.

As indicated in the introduction, we model the beam as a Gaussian beam with the corresponding transverse irradiance profile ${ }^{13}$ and spatial divergence. ${ }^{16,17}$ Using the above definition of the beam coordinate system and assuming further that the beam waist is at $\zeta=0$, the irradiance at a point $P_{i}$ in space can be calculated from

$$
E\left(\rho_{i}, \zeta_{i}\right)=E_{0} \frac{w_{0}^{2}}{w\left(\zeta_{i}\right)^{2}} \exp \left(-\frac{2 \rho_{i}^{2}}{w\left(\zeta_{i}\right)^{2}}\right)
$$

where $\zeta_{i}$ is the $\zeta$-coordinate of the point (i.e., its distance from the beam waist, measured along the beam center), $\rho_{i}=\sqrt{\xi_{i}^{2}+\eta_{i}^{2}}$ is its distance from the beam center, $E_{0}$ is the irradiance at the center of the beam waist, and $w\left(\zeta_{i}\right)$ is the beam radius at $\zeta_{i}$. The beam radius, in turn, depends on the wavelength $\lambda$, the beam waist radius and the distance:

$$
w(\zeta)=w_{0} \sqrt{1+\left(\frac{\lambda \zeta}{\pi w_{0}^{2}}\right)^{2}}
$$

Different definitions can be used for the beam width depending on the encircled power. ${ }^{18}$ The above beam radius $w(\zeta)$ corresponds to the distance from the beam center at which the irradiance has dropped off to $13.5 \%$ of its peak value $\left(\frac{1}{e^{2}}\right)$. Thus a perpendicular cross section of the beam, restricted to a circle with radius $w(\zeta)$ contains $86 \%$ of the total radiated beam power at the corresponding distance. For the later integration over the entire footprint (see Eq. 1a) we use the beam divergence half-angle

$$
\gamma_{0}=\frac{\lambda}{\pi w_{0}}
$$

instead of the beam radius to define the integration limits and thus the effective border of the footprint. In particular we integrate over a solid angle corresponding to $4 \gamma_{0}$ i.e., $2 k \gamma_{0}$ with $k=2$ (cf. Figure 1 ). Assuming that the measured surfaces are far enough from the scanner such that the non-zero diameter of the beam at the 
scanner and the non-linear dependence of the beam diameter on distance near the beam waist can be ignored, this corresponds to integration over an area containing $99.99 \%$ of the total radiated energy within the beam.

We represent the reflecting surfaces by triangular irregular meshes (TINs) of high spatial resolution. Each triangle is associated with material properties, currently only representing the (constant) reflectivity of the triangular surface patch according to a purely diffuse Lambertian model. ${ }^{7,19}$ The size of the triangles in this representation can be chosen smaller than the footprint. This allows properly representing spatial variations of material properties or of geometry within the footprint.

For the simulation of the scanning process, the beam center direction $\left(\theta_{0}, \phi_{0}\right)$ is incrementally defined for each point to be scanned. Along with the above definition of the Gaussian beam the irradiance distribution in the $3 \mathrm{~d}$ space of the SOCS is then given for each measurement. We discretize the beam into rays defined by a regular grid of solid angles arranged symmetrically about the beam center. The optical power associated with each ray is calculated from the irradiance $E_{i}$ at the intersection point of the ray with the TIN, assuming constant irradiance within a small neighborhood about the ray. This neighborhood with area $A_{i}$ on the surface is delimited by a polygon resulting from the intersection of four lines with the surface. The lines delimit a space symmetric about the ray and extending by half the angular discretization increment in the direction of both $\theta$ and $\phi$.

A high degree of discretization (approximately $60 \mathrm{k}$ rays in each beam) is chosen such that even minute surface features are taken into consideration. Consequently, the area on the surface over which the power is computed for an individual ray is very small. For simplicity possible intersections of the polygons and the edges of the triangles within the TIN are ignored, and the power is calculated assuming the entire area $A_{i}$ to be part of the triangle intersected by the respective ray. We verified numerically that this approximation has negligible effect on the accuracy of simulated distance measurement, if the triangles are much smaller than the footprint and the surface is sufficiently homogeneous within the footprint. An investigation of the limitations of this approach and potential improvement of the approximation for surfaces with significant roughness or variability of the material properties is left for future work.

The incident power $P_{o, i}$ at the area associated to the $i^{t h}$ ray at the surface is given by

$$
P_{o, i}=E_{i} \cdot A_{i} \cdot \cos \left(\alpha_{i}\right),
$$

where $\alpha_{i}$ denotes the angle of incidence at the intersection point (see Figure 1 for the ray along the beam center). The reflected power $P_{r, i}$ is then calculated for each ray considering the reflectance $R$ associated to the corresponding surface patch as

$$
P_{r, i}=P_{o, i} \cdot R \text {. }
$$

To compute the power reaching the detector after diffuse reflection, the Lidar equation ${ }^{20,21}$ is implemented. According to the reflection model of the surface (pure diffuse Lambertian) allocated with each triangle, the maximum optical intensity $I_{r, \max }$ after reflection is obtained in the normal direction. It relates to the total radiated power as

$$
I_{r, \max }=\frac{P_{r, i}}{\pi},
$$

and decreases with the cosine of the viewing angle. Noting that the emitter and receiver optics are typically collocated and coaligned in this kind of instruments, this viewing angle coincides with the angle of incidence $\alpha_{i}$ of the corresponding ray at the surface. The optical intensity in the direction of the detector is therefore

$$
I_{r, i}=I_{r, \max } \cdot \cos \left(\alpha_{i}\right) .
$$

The power $P_{D, i}$ received at the detector is in turn calculated by accounting for the effective field of view of the detection unit, i.e, by considering the area $\left(A_{D}\right)$ of the detector and its angular orientation $\left(\alpha_{D}\right)$, following:

$$
P_{D, i}=I_{r, i} \cdot \frac{A_{D} \cos \left(\alpha_{D}\right)}{d_{i}^{2}}
$$

where $d_{i}$ is the geometrical distance for the $i_{t h}$ ray between its intersection point on the surface and the detector. 
The detected power $P_{D, i}$ acts as a weighting factor for the phase $\left(\Phi_{i}\right)$ associated to each ray in the final distance estimation. This phase is calculated from $d_{i}$ taking into account the scale introduced by the modulation frequency $\left(\nu_{m}\right)$ of the laser as

$$
\Phi_{i}=\frac{2 \cdot d_{i} \cdot 2 \pi \cdot \nu_{m} \cdot n_{g}(\lambda)}{c}
$$

with $c$ being the propagation speed of light in vacuum and $n_{g}(\lambda)$ the group refractive index of air at the center optical wavelength $\lambda$ of the laser. The refractive index is in practice calculated from meteorological data representing the density of the air. ${ }^{22}$ For the simulation purposes it is sufficient to assume standard air and standard atmospheric conditions.

Once the detected power and phase are computed for all the rays within the measurement beam, the total phase $\hat{\Phi}_{T}$ is calculated via I/Q demodulation. ${ }^{12}$ The simulated I- and Q-components, corresponding to the cos and $\sin$ of $\hat{\Phi}_{T}$, respectively, are obtained by integration of the weighted $\cos \Phi_{i}$ and $\sin \Phi_{i}$ over all the rays of the footprint. The weights correspond to the received power from the surface area $A_{i}$ of the respective ray. Within the numerical simulation, the integration can be replaced by summation over all rays:

$$
\hat{\Phi}_{T}=\arctan \left(\frac{\sum P_{D, i} \cdot \sin \left(\Phi_{i}\right)}{\sum P_{D, i} \cdot \cos \left(\Phi_{i}\right)}\right)
$$

Finally, the estimated distance $\hat{D}$ corresponding to a particular beam direction $\left(\theta_{0}, \phi_{0}\right)$ and a particular modulation wavelength $\lambda_{m}$ is derived from the total phase according to the following equation

$$
\hat{D}\left(\theta_{0}, \phi_{0}, \lambda_{m}\right)=\hat{\Phi}_{T} \cdot \frac{\lambda_{m}}{4 \pi}+N_{m} \cdot \lambda_{m}+e_{m}
$$

with a simulated random deviation $e_{m}$ representing noise, and a distance ambiguity determined by an unknown integer multiple $\left(N_{m}\right)$ of the modulation wavelength

$$
\lambda_{m}=\frac{c}{\nu_{m} \cdot n_{g}} .
$$

The ambiguity occurs because the phase-based measurement only yields phase angles within $[0,2 \pi)$ as can be seen from Eq. 12, and is not sensitive to changes of the distance by integer multiples of the modulation wavelength. Within this paper, we will not address the problem of ambiguity resolution, i.e. of determining the value of $N_{m}$ which is practically done by measuring using different modulation frequencies simultaneously, and we will not address actual simulation of random measurement noise $e_{m}$. Instead we consider herein, that $e_{m}=0$ and that the modulation wavelength is longer than twice the maximum measurement distance such that necessarily $N_{m}=0$. Furthermore, we will not write the two angles characterizing the direction of the beam unless we need to distinguish different beams. So we finally have

$$
\hat{D}=\hat{D}\left(\theta_{0}, \phi_{0}\right)=\hat{\Phi}_{T} \cdot \frac{\lambda_{m}}{4 \pi}
$$

We define the distance error $(\epsilon)$ of the (simulated) measurement as the deviation from the true distance $\left(d_{0}\right)$ of the intersection point of the beam center axis:

$$
\epsilon:=\hat{D}-d_{0}
$$

Based on the above calculation of the distance measurement and error of an individual beam, the 3d laser scanning process can readily be simulated by deflecting the beam into other directions. The simulation can be parallelized for computational efficiency, since the calculations can be carried out separately for each beam. We will use this approach in Section 3 to carry out analysis of distance biases for some geometrically and radiometrically simple situations. However, the approach can be easily extended to take non-Lambertian reflection or other effects like surface penetration into account by modifying the calculation of the received power or adding distance biases in Eq. 11 depending on material, angle-of-incidence, and possibly further parameters. Such an extension is left for future work. 


\subsection{Experimental investigations}

Within this paper experimental investigations are used for a first validation of the numerical simulations. The experiments were conducted in our geodetic metrology lab under controlled, known and reproducible conditions. The concept of the laboratory measurements is depicted in Figure 3. A laser scanner is set up on a stable pillar on one end of support bench. A target specimen is mounted on the bench at a chosen, fixed distance from the scanner. A background plane, significantly larger than the target specimen, is mounted at an edge of a trolley which can be moved along the bench. However, it cannot get closer to the scanner than the target specimen. The plane is kept approximately vertically such that the angles of incidence are nearly orthogonal. The setup also includes a laser interferometer which can be used to measure the true displacement of the trolley (i.e. the background plane) when it is moved along the supporting bench.

By scanning the target, the effect of target surface curvature and angle of incidence can be investigated. By placing the background plane at different distances, and by using planes of different reflectivity as background, the effect of mixed pixels close to the edges of the target specimen can be investigated.

In the concrete analyses used subsequently, the numerical simulations and the experiments have been designed such as to correspond well to each other. In particular, we have used a cardboard cylinder of $9.3 \mathrm{~cm}$ diameter as target specimen. We mounted it on a pillar at a distance of approximately $17.40 \mathrm{~m}$ from the scanner, with its longitudinal axis approximately vertically. A highly reflective and a less reflective background plane were used (Figure 4). The scans were made using a phase-based $\mathrm{Z}+\mathrm{F}$ imager 5016, and the parameters for the simulations were chosen accordingly.

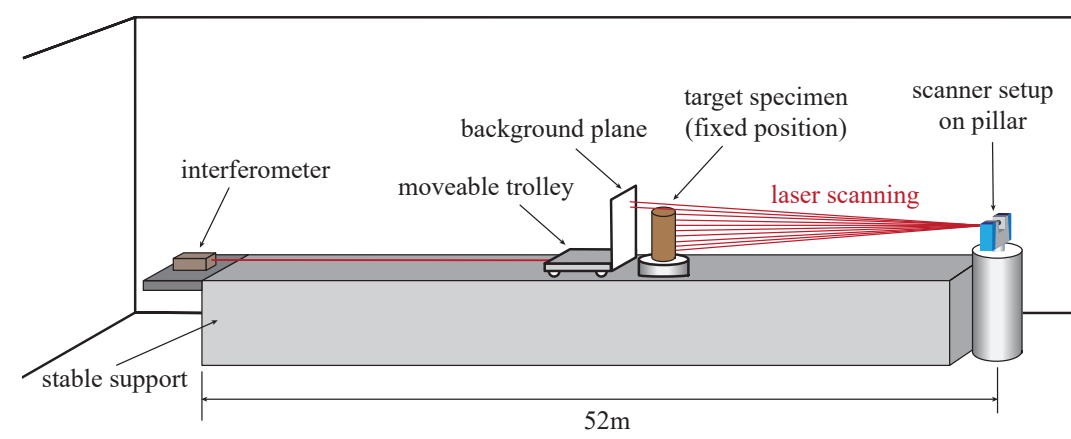

Figure 3: Concept diagram of lab experiments.

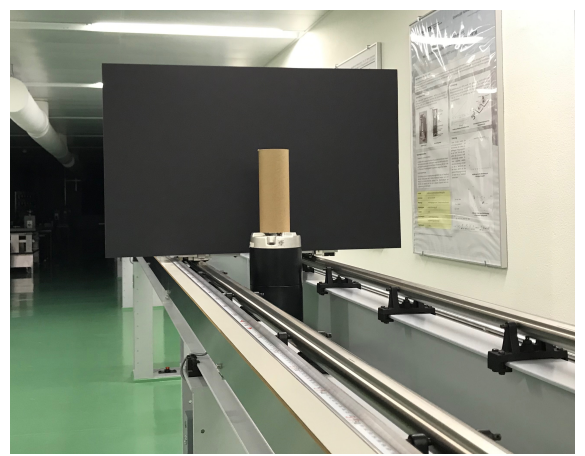

(a) Dark background

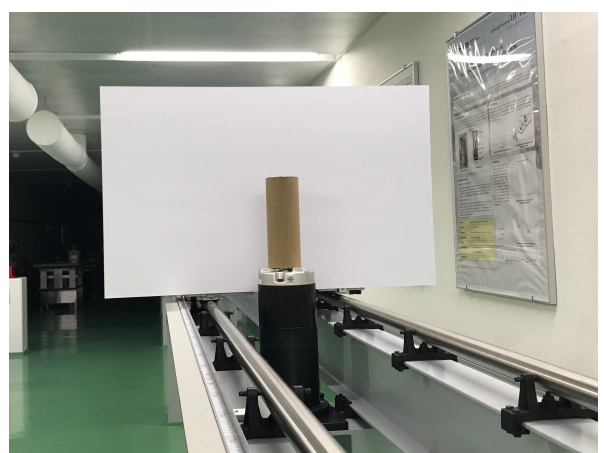

(b) Bright background

Figure 4: Laboratory experimental setup showing the cylinder and the two background planes. 


\section{RESULTS}

\subsection{Tilted plane}

First we investigate whether there is a distance bias due to the angle of incidence of the laser beam when measuring onto a homogeneous, perfectly planar surface (see Figure 1). Typical beam waist radii of terrestrial laser scanners are on the order of a few mm according to the specifications, and the beam divergence half-angles are on the order of a few $0.1 \mathrm{mrad}$. The numerical simulations were thus carried out with beam waist radii of (a) $w_{0}=1 \mathrm{~mm}$ and (b) $w_{0}=4 \mathrm{~mm}$ for a laser with a wavelength of $1550 \mathrm{~nm}$. Figure 5 shows the resulting distance biases $\epsilon$ for angles of incidence between 0 (orthogonal beam) and $80 \mathrm{deg}$, and for four different true distances $d_{0}$ from 10 to $300 \mathrm{~m}$.

In comparison to the typical noise level of TLS measurements (a few $\mathrm{mm}$ ) and the anticipated effects of surface roughness, the distance biases predicted by this simulation are negligible. They stay below $0.1 \mathrm{~mm}$ except for relatively large distances and incidence angles with the beam corresponding to the smaller waist. It may be surprising to see that the larger beam waist (b) is associated with even lower biases. However, this is due to the fact that the beam divergence is inversely proportional to the beam waist radius (see Eq. 5). In the specific case shown here, the beam diameters are equal at about $8 \mathrm{~m}$ distance from the beam waist, with the beam diameter of beam (a) growing four times as rapidly as that of (b). Thus the variation of distances within the footprint is much bigger for beam (a) except at very short distances.

A detail, interesting from the theoretical point of view, is the small positive bias if the beam strikes the surface orthogonally $\left(\alpha_{i}=\right.$ 0degrees). This occurs because all the rays within the footprint, except the center ray, have a longer distance than the true one $\left(d_{0}\right)$ in this case. However, with increasing angle of incidence the closer surface parts start contributing more to the measurement value (mainly because of the Lambertian reflection model assumed herein) and thus the bias is negative at large angles of incidence.

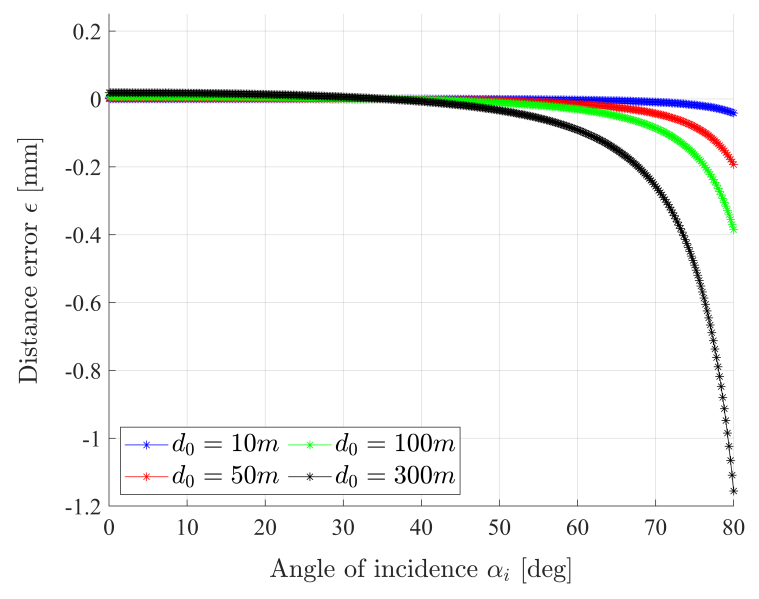

(a) $w_{0}=1 \mathrm{~mm} ; \gamma_{0}=0.49 \mathrm{mrad}$

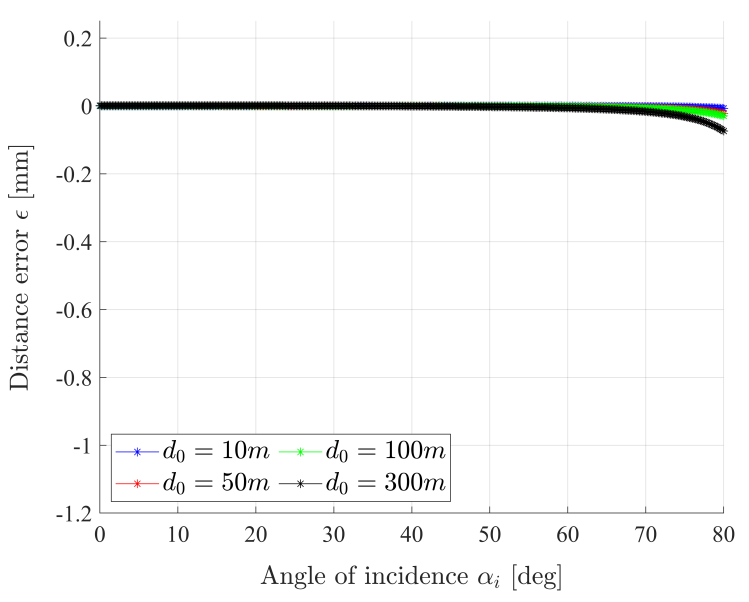

(b) $w_{0}=4 \mathrm{~mm} ; \gamma_{0}=0.12 \mathrm{mrad}$

Figure 5: Bias of distance measurement onto a planar homogeneous surface for different distances, different beams, and varying angle of incidence.

\subsection{Cylinder with planar background}

The simulation and laboratory experiments were also carried out for a cylinder as target, in front of a background with $5 \%$ reflectance and one with $95 \%$ (see Figure 4). Additionally a case with non-reflecting background $(0 \%$ reflectance) was simulated. The diameter and height of the cylinder, the distances and the reflectances were chosen similarly in the experiment and in the numerical simulation. Moreover, the laser beam parameters were selected according to the spec-sheet of the laser scanner which reports a beam divergence half-angle (ref. Eq. 5) of $0.3 \mathrm{mrad}$ and a beam diameter $\left(\frac{1}{e^{2}}\right)$ of about $3.5 \mathrm{~mm}$ at $1 \mathrm{~m}$ from the scanner. Based on these specifications, 
the beam profile of the Gaussian $\mathrm{TEM}_{00}$ beam was assumed. For the simulated signal superposition and I/Qdemodulation according to Section 2.1 a modulation wavelength of $100 \mathrm{kHz}$ was chosen. While this value was significantly lower than the actual short-wavelength modulation frequency of the real scanner (not known from the specification, mixed pixel effects suggest a value of slightly above $200 \mathrm{MHz}$ ), the cylinder and the background were within the unambiguity range for all cases investigated herein, and measurement noise was not simulated. Therefore, the concrete choice of modulation wavelength for the simulation was not critical.

The simulations and experiments are intended to study the impact of surface curvature within the footprint (cf. beam A in Figure 6, with the footprint entirely on the surface of the cylinder) and the impact of mixed pixels (cf. beam B in the figure, with the beam only partially hitting the cylinder).

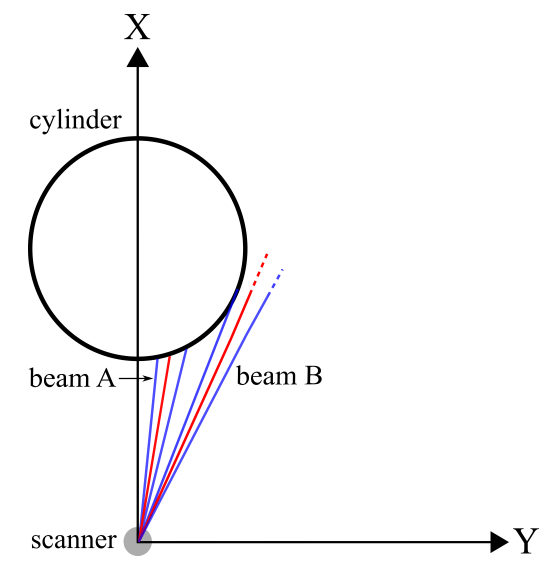

Figure 6: Schematic diagram showing the laser beam hitting or partially missing the cylinder surface.

Figure 7 shows horizontal cross sections of the point clouds obtained from the simulation, and a sketch of the location of the horizontal cross-sections in relation to the entire cylinder. The reflectance of the cylinder was assumed to be $55 \%$. The cross sections plotted on the left are obtained at a height where the laser beam towards the middle of the cylinder intersects the surface nearly orthogonally. In this case, the point clouds obtained with the 95\%-, 5\%- and $0 \%$ background, respectively, coincide up to a certain scanning angle from the middle of the cylinder. For these points, the footprint is entirely on the cylinder surface (beam of type A) and thus the background is irrelevant for the measurement result. Closer to the edges of the cylinder, the beam lands partially on the cylinder surface and partially on the background (beam of type B) which gives rise to mixed pixels. The resulting errors depend on the exact location of the footprint w.r.t. the edge, on the distances, on the curvature, and - above all — on the the relative reflectance of foreground (cylinder) and background.

The points when the signal is mixed with strong reflecting background tend to be closer to the cylinder surface compared to the points simulated when the background is dark (less reflective). With the brighter background, mixed pixels are already discernible closer to the middle of the cylinder than with the darker background. This is in correspondence with the thought model of the distance measurement being some weighted average of the distances within the footprint, with the weights depending on the power received from the different surfaces.

The pattern of the biases is markedly different for the $0 \%$-reflectance of the background (black circles). In this case, the bias is not due to actual mixed pixels but to the fact that the distance measurement only refers to an edge of the footprint while the angles $\left(\theta_{0}, \phi_{0}\right)$ measured by the scanner and used to calculate the Cartesian coordinates of the scanned points refers to the beam center. These biases can be seen as a limiting case also for the mixed pixels scenario, indicating what is to be expected if the background reflection is negligible, e.g. because the background is far away. If a circle is fitted to such data e.g., in order to determine the diameter of the cylindrical object, ${ }^{14}$ it is to be expected that the diameter is significantly overestimated unless the points towards the edges are identified as outliers and excluded from the estimation process.

The biases shown in Figure 7(b) refer to the horizontal cross section at the top of the cylinder. Here, all points with reflecting background are affected by mixed pixels, and again, the magnitude of the bias clearly shows that the brighter background has much bigger impact. 


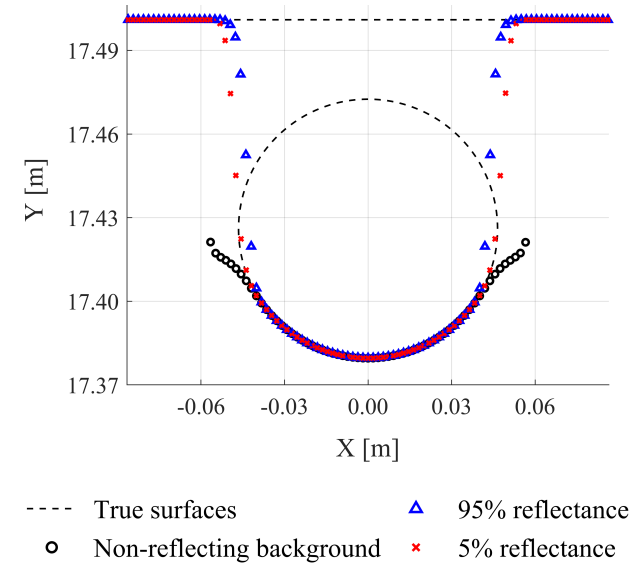

(a) Orthogonal cross-section profile

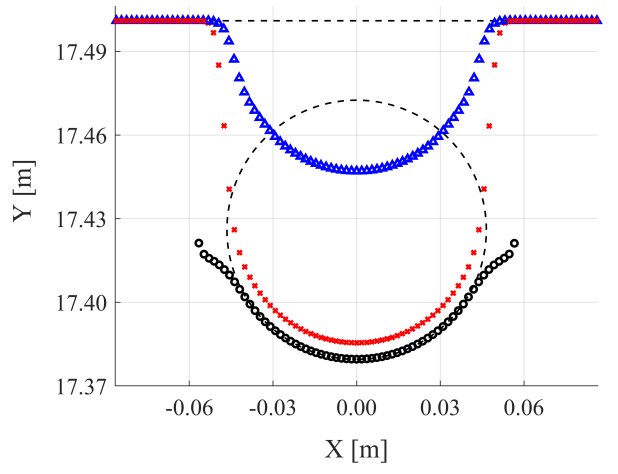

$\begin{array}{ccc}\text {--- True surfaces } & \Delta & 95 \% \text { reflectance } \\ \circ \quad \text { Non-reflecting background } & \times & 5 \% \text { reflectance }\end{array}$

(b) Top cross-section profile

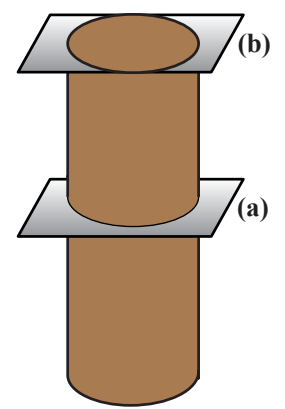

(c) Cross-section profiles

Figure 7: Horizontal cross-sections of the cylinder, the planar background and three simulated point clouds corresponding to varying background reflectance (see legend).

Results from the laboratory measurement are shown in Figure 8. A horizontal profile is taken at a height corresponding to Figure 7(a). The mixed pixel effects follow a similar pattern to the ones predicted by the simulation and thus corroborate the simulation results qualitatively and in terms of order of magnitude. Since the real measurements are also affected by noise, the real cylinder is slightly deformed (mm-level deviations from a perfect cylinder), does not act like a perfectly homogeneous Lambertian reflector, and the actual beam profile is not perfectly Gaussian $\mathrm{TEM}_{00}$, the results cannot be exactly the same. Furthermore, a sufficiently absorbing background to resemble the 0\%-reflectance case from the simulation was not available for comparison.

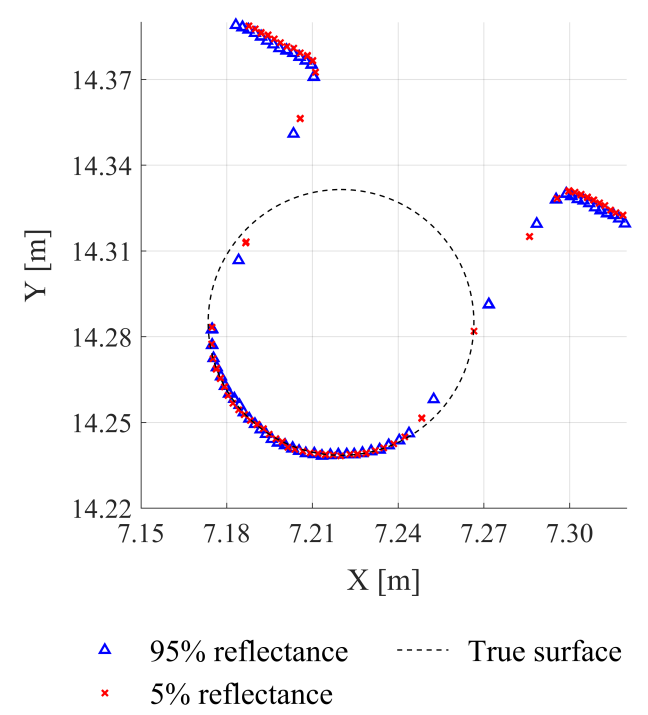

Figure 8: The point clouds acquired from the laboratory experiments.

The effect of the surface curvature, the angle of incidence and the mixed pixels for the scans of the cylinder can be seen more clearly in Figure 9 where the distance bias is plotted against the angle of incidence for only beams whose center ray intersects the cylinder, i.e. beams of type A (cf. Figure 6) and beams of type B with 
more than $50 \%$ of the footprint still on the cylinder. The data are taken from the previous simulation with $0 \%$-reflectance of the background. The dotted red line at $51.35 \mathrm{deg}$ shows the largest angle of incidence for which the footprint is still entirely on the cylinder. The errors to the left of the dotted line show the combined effect of the surface curvature and the angle of incidence; they are at the order of magnitude of the effects found in Section 3.1 for the plane, i.e. less than $0.1 \mathrm{~mm}$ and thus practically most likely negligible in real scanning applications with noise and rough surfaces. However, the rapid increase of the biases to the right of the red line indicates the significant effect of beams partially missing the object. The related errors in the mixed pixels case, i.e., with significantly reflecting background, are even larger and can reach practically significant levels even with a significant part of the beam still hitting the object.

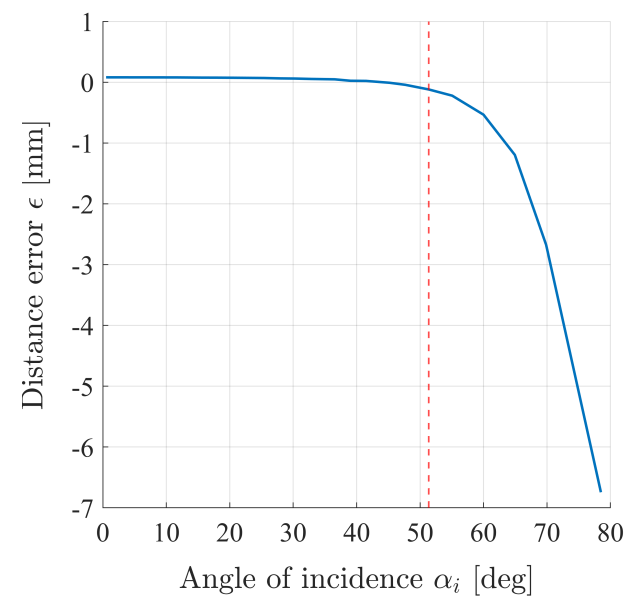

Figure 9: The distance error $(\epsilon)$ for the cylindrical surface plotted against angle of incidence $\left(\alpha_{i}\right)$.

\section{CONCLUSIONS}

We presented a versatile simulation framework for modeling the LiDAR measurement process assuming phasebased reflectorless electronic distance measurement (RL-EDM) with I/Q-demodulation and a Gaussian beam profile. By taking into account the deflection of the laser beam at incremental angular intervals the framework was extended to one of the $3 \mathrm{~d}$ laser scanning process. The object surfaces are represented by triangulated irregular networks with triangles which can be small in relation to the footprint of the laser. In combination with the association of each triangle with material parameters, this allows simulating measurements affected by geometric or radiometric changes within the footprint. The four major steps of the presented framework are:

1. Definition of the surface and surface parameters.

2. Definition of the laser beam parameters.

3. Discretization of the laser beam into a fixed number of rays intersecting each of them with the surfaces and calculating the corresponding contribution to the total power and phase at the detector.

4. Estimation of the distance bias by retrieving the distance estimate from the total phase after I/Q demodulation, and subtraction of the true distance along the beam center.

Herein we have applied the simulation to investigate the distance biases due to the angle of incidence on a planar reflector, and due to angle of incidence, surface curvature and mixed pixels for measurements onto a cylindrical surface. We found that the angle of incidence can cause positive or negative biases at the order of $0.1 \mathrm{~mm}$ or less except for very large angles of incidence and large footprints, in which case the bias can reach mm level. However, these biases are much smaller than the typical noise levels of the measurements or the effects of surface roughness, such that they are practically negligible. The same holds for the surface curvature effects 
found with the cylindrical target. On the other hand, the simulations clearly showed the significant impact of mixed pixels effects and of errors arising if only a part of the beam hits the object surface. These are dominant errors also affecting real point clouds.

We verified the simulation results using real scans obtained in a laboratory. The experimental results corroborated the numerical simulations qualitatively and in terms of order of magnitude. The remaining quantitative discrepancies indicate that the simulation approach will have to be extended to predict the real biases with higher accuracy. The intended extensions refer to the implementation of more realistic beam profiles, a more flexible reflectivity model, surface roughness and sub-surface reflections. With those extensions, the simulation framework is a useful step towards a numerical model for the practically useful quantification of surface-related uncertainties in terrestrial laser scanning.

\section{ACKNOWLEDGMENTS}

This work was supported by the Swiss National Science Foundation (SNSF) under grant number 169318. R. Presl is acknowledged for providing Figure 2.

\section{REFERENCES}

[1] Vosselman, G. and Maas, H., [Airborne and Terrestrial Laser Scanning], Whittles Publishing (2010).

[2] Schulz, T., Calibration of a terrestrial laser scanner for engineering geodesy, PhD thesis, ETH Zurich (2008).

[3] Reshetyuk, Y., Self-calibration and direct georeferencing in terrestrial laser scanning, PhD thesis, KTH, Geodesy (2009). QC 20100806.

[4] Zámeníková, M., Wieser, A., Woschitz, H., and Ressl, C., "Influence of surface reflectivity on reflectorless electronic distance measurement and terrestrial laser scanning," Journal of applied geodesy 8(4), 311-325 (2014).

[5] Zámečníková, M., Neuner, H., Pegritz, S., and Sonnleitner, R., "Investigation on the influence of the incidence angle on the reflectorless distance measurement of a terrestrial laser scanner," Iulia Dana Negula, Violeta Poenaru, 37.

[6] Gordon, B., Zur Bestimmung von Messunsicherheiten terrestrischer Laserscanner, PhD thesis, Technische Universität (2008).

[7] Soudarissanane, S. S., "The geometry of terrestrial laser scanning: Identification of errors, modeling and mitigation of scanning geometry," (2016).

[8] Koch, K. R., Kuhlmann, H., and Schuh, W.-D., "Approximating covariance matrices estimated in multivariate models by estimated auto- and cross-covariances," Journal of Geodesy 84, 383-397 (Jun 2010).

[9] Wujanz, D., Burger, M., Mettenleiter, M., and Neitzel, F., "An intensity-based stochastic model for terrestrial laser scanners," ISPRS Journal of Photogrammetry and Remote Sensing 125, 146-155 (2017).

[10] Lichti, D. D. and Jamtsho, S., "Angular resolution of terrestrial laser scanners," The Photogrammetric Record 21(114), 141-160 (2006).

[11] Karp, S. and Stotts, L. B., [Fundamentals of Electro-Optic Systems Design: Communications, Lidar, and Imaging], Cambridge University Press (2012).

[12] Sha, Z., Feng, H., and Zeng, Z., "Phase demodulation method in phase-sensitive otdr without coherent detection," Opt. Express 25, 4831-4844 (Mar 2017).

[13] Milonni, P. and Eberly, J., [Laser Physics], Wiley (2010).

[14] Forsman, M., Brlin, N., Olofsson, K., Reese, H., and Holmgren, J., "Bias of cylinder diameter estimation from ground-based laser scanners with different beam widths: A simulation study," ISPRS Journal of Photogrammetry and Remote Sensing 135, 84 - 92 (2018).

[15] Wagner, W., Ulrich, A., Melzer, T., Briese, C., and Kraus, K., "From single-pulse to full-waveform airborne laser scanners: Potential and practical challenges," Int. Archives Photogrammetry, Remote Sensing, Geoinformation Sci. 35, 414-419 (01 2004).

[16] Saleh, B. and Teich, M., [Fundamentals of Photonics], Wiley Series in Pure and Applied Optics, Wiley (2019).

[17] Siegman, A. E., "New developments in laser resonators," (1990). 
[18] Hodgson, N. and Weber, H., [Laser Resonators and Beam Propagation: Fundamentals, Advanced Concepts, Applications], Springer Series in Optical Sciences, Springer Berlin Heidelberg (2014).

[19] Rees, W. G., [Physical Principles of Remote Sensing], Scott Polar Research Institute, Cambridge (2001).

[20] Weitkamp, C., [Lidar: Range-Resolved Optical Remote Sensing of the Atmosphere], Springer Series in Optical Sciences, Springer New York (2006).

[21] Pfeifer, N., Höfle, B., Briese, C., Rutzinger, M., and Haring, A., "Analysis of the backscattered energy in terrestrial laser scanning data," in [ISPRS 2008: Proceedings of the XXI congress : Silk road for information from imagery : the International Society for Photogrammetry and Remote Sensing, 3-11 July, Beijing, China. Vol. XXXVII, Comm. V, Part B 5-2, pp. 1045-1051], XXXVII, 1045-1051, International Society for Photogrammetry and Remote Sensing (ISPRS) (2008).

[22] Ciddor, P. E. and Hill, R. J., "Refractive index of air. 2. group index," Applied Optics 38(9), 1663-1667 (1999). 